\title{
UTILISATION PRATIQUE DE LA METHODE DE SIMULATION DANS L'ASSURANCE ,NON LIFE”
}

\author{
D'Hooge, Franckx et Gennart \\ Bruxelles \\ I. - InTRODUCtion
}

Les colloques successifs d'Astin ent eu le mérite de clarifier certaines idées théoriques importantes. En invitant au colloque d'Arnhem les actuaires à penser aux méthodes de Monte Carlo, les organisateurs ont forcément orienté les recherches vers le domaine des applications. Car simuler, c'est à dire établir des résultats conformes à un processus aléatoire bien déterminé, suppose à priori que les données numériques d'introduction (ou de définition) ont été fixées.

Nous ne reprendrons pas les principes de la méthode de simulation, ils sont donnés de manière magistrale dans „Notes on operations research I959" du centre de recherche opérationnelle du Massachusetts Institute of Technology. Le chapitre II, portant titre "Simulation of Random processes" rédigé par Herbert P. Galliher donne un exposé complet du problème. Nous renvoyons à cette référence.

Parmi les idées que l'on doit retenir, car elle est d'application dans tous les domaines, nous citons "Never simulate a single numerical problem;always simulate several of a class of problems".

Dans le domaine non life, cet aspect est fondamental. Ce qui y fait défaut, ce sont des algorithmes généraux de résolution numériques. Une des difficultés majeures rencontrées par les actuaires a été et est encore le calcul effectif des convolutions. Ceux qui ont établi cette note se sont posé la question de savoir si la méthode de Monte Carlo ne pouvait fournir un procédé:

- évitant les convolutions.

- suffisamment général pour être applicable à une classe de risques arbitraire.

- donnant comme sous-produit des éléments intéressant aussi bien les théoriciens que les praticiens. 
Une réponse largement positive semble donnée à ces desiderata.

La base de la méthode de simulation est le théorème de CantelliGlivenko: ,lorsque la taille d'un échantillon augmente la fonction de distribution de l'échantillon converge fortement vers la fonction de distribution de la population dont l'échantillon a été extrait".

Un risque quelconque - n'appartenant pas nécessairement à une classe homogène - est défini par deux coordonnées stochastiques. D'une part l'intensité $d u$ risque (valeur aléatoire du nombre de sinistres frappant un contrat au cours de l'année), d'autre part la hauteur $d u$ sinistre (montant aléatoire de chaque sinistre particulier). L'algorithme de simulation doit être programmé de façon à être utilisable quelles que soient les coordonnées stochastiques.

En introduisant dans l'ordinateur des valeurs particulières - donc numériques - de ces coordonnées stochastiques, celui-ci réalisera une épreuve simulée, dont le résultat est la dépense totale annuelle pour un contrat. En répétant cette épreuve un grand nombre de fois, et en enregistrant les résultats, on constitue la population numérique de référence. A partir de cette dernière, on déduira comme sous-produits par des sous-routines les autres résultats statistiques. Dans nos expériences, cette population de base comportant 360.000 valeurs simulées (nombre non pris au hasard), elle nous a permis de nous rendre compte de la convergence effective annoncée par le théorème de Cantelli-Glivenko.

Le métier d'assureur est possible parce que par les cumuls des résultats individuels des régularités statistiques sont constatées. Le problème des cumuls est par suite essentiel.

Une question que nous nous sommes posée peut s'énoncer comme suit: Existe-t-il une méthode systématique permettant de calculer le budget stochastique relatif à un nombre arbitraire de contrats?

En d'autres mots, si un ensemble de risques comporte $k=765$ contrats est il possible de donner en début d'exercice les valeurs numériques de la fonction de distribution du total à payer pour cet ensemble de contrats?

Deux méthodes peuvent être envisagées:

- la première: par cumul des résultats de contrats de la population de base et application de la méthode de Cantelli-Glivenko; elle a 
été effectivement utilisée pour les valeurs de $k=1,4,9,25$, 100, 400, 900 .

- la seconde: par une méthode d'approximation, (interpolation ou extrapolation) à partir de certaines courbes de budget particulières (celles déduites par la première méthode) on cherche à établir celle de l'effectif imposé. La deuxième méthode est basée sur l'utilisation des „,courbes de transformations". Si $F(x)$ et $\Phi(y)$ sont deux fonctions de répartition des variables $X$ et $Y$, une telle courbe de transformation définit la fonction $y=f(x)$ telle que: $\Phi[f(x)] \equiv F(x)$

Dans le cadre d'une étude numérique, l'image statistique des courbes de transformation est obtenue à la machine, mais ajustée graphiquement ou par calcul élémentaire. Le choix de la fonction de référence $F(x)$ n'est pas arbitraire. L'idée d'Esscher nous a amené à essayer la loi normale en particulier.

Des résultats encourageants ont été obtenus de cette manière, ils indiquent que des études subséquentes sont souhaitables.

A partir du moment où l'on dispose du budget stochastique d'un ensemble de risques, on peut étudier tous les problèmes et établir n'importe quelle tarification. Dans cette note, nous avons voulu étudier.

- l'influence de l'effectif d'une classe de risques sur les chances qu'a un assureur de subir des fluctuations défavorables.

- l'influence de l'effectif d'une classe de risques sur la partition de la prime globale pure entre assureur er réassureur, lorsque ce dernier prend à sa charge l'indemnisation au delà d'une certaine limite de retention (stop loss).

Ce choix particulier montre que les études ont atteint actuellement un niveau tel que la résolution effective et numérique des problèmes pratiques est devenu possible.

Cependant, pour que l'algorithme soit général, et les résultats comparables, il a fallu prendre une définition invariante des fluctuations défayorables. Si $E$ désigne la valeur moyenne du budget d'une classe de risques, une fluctuation défavorable sera mesurée par:

$$
\begin{gathered}
y=E(\mathrm{x}+\rho) \\
\rho \geqslant 0
\end{gathered}
$$


La fluctuation $y$ correspond à une dépense budgétaire dépassant la valeur probable. La valeur de $E$ dépend des coordonées stochastiques et de l'effectif qui varient de cas en cas.

Dès lors, le paramètre principal de l'étude est la valeur de $\rho$, exprimée en pour cents de la valeur moyenne.

L'influence de l'effectif est non négligeable; les résultats consignés dans des tableaux permettent les calculs réels pour un effectif quelconque. Un rapprochement avec les considérations de la fullcredibility américaine est instructif.

Le présent travail a été exécuté en team. L'un d'entre nous s'est chargé de la conception générale, le second de la programmation et de l'exécution sur IBM I620, le troisième de l'exploitation des résultats. Ce team a eu le sentiment net d'avoir appris beaucoup dans l'exécution du présent travail. Il espère que cette expérience sera aussi constructive pour les autres.

\section{Description des travaux de simulation efFectués}

\subsection{Données}

a - La variable aléatoire $N$, intensité $d u$ risque, suit une loi dont la fonction de répartition est une image statistique observée, suivant les données ci-après

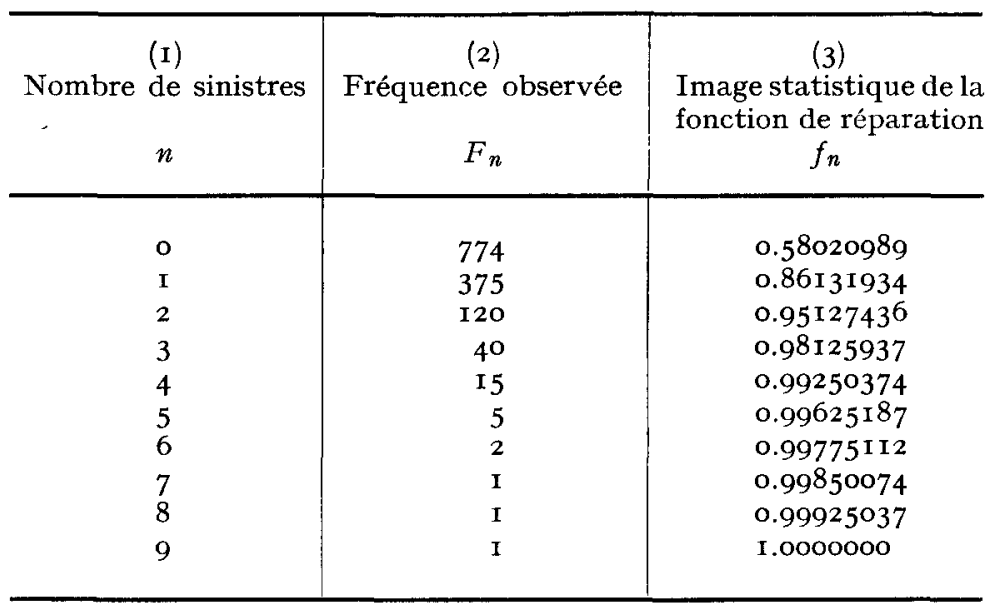


Cette loi est extraite de l'article: „Un problème de tarification de l'assurance accidents d'automobiles examiné par la statistique mathématique" Pierre Delaporte, XVIe Congrès International d'Actuaires. Vol. II, Ig6o.

b - La variable aléatoire $M$, hauteur d'un sinistre, suit la loi théorique exponentielle de moyenne unité dont la fonction de réparation est

$$
F(m)=\operatorname{Prob}(M<m)=\mathrm{I}-e^{-m}
$$

c - Pendant la période de référence considérée (une année), un contrat subit un nombre $n$ de sinistres, dont la somme des dégats

$$
S=m_{1}+m_{2}+\ldots+m_{n}
$$

est également une variable aléatoire et représente la dépense pośsible de l'assureur pour le contrat considéré.

\subsection{Création d'un fichier de base}

On a simulé 360.000 contrats pendant la période de référence d'une année. On a noté pour chacun d'entre eux les valeurs obtenues pour les variables $N$ (intensité) et $S$ (somme des dégats). Ces résultats ont été perforés sur des cartes à raison de 6 contrats par carte, et ont servi à l'établissement des statistiques décrites dans les paragraphes suivants.

La simulation d'un contrat se fait par la méthode classique suivante. La génération d'une variable aléatoire de fonction de répartition donnée se fait à partir de nombres pseudo-aléatoires à distribution uniforme sur l'intervalle $(0, I)$.

Pour ceux-ci, on utilise les formules suivantes (nombres entiers):

$$
\begin{aligned}
X_{1}=40353607=7^{9} & \\
X_{j+1}=a X_{j}(\bmod M) \text { avec } a & =7^{9} \\
M & =10^{10}
\end{aligned}
$$

Les nombres pseudo-aléatoires (compris entre o et $\mathrm{I}$ ) sont

$$
x_{j}=\mathrm{I0}^{-10} X_{j} \quad j=\mathrm{I}, 2, \ldots
$$

Chaque fois qu'on a besoin d'un tel nombre, quel que soit son usage, on le détermine à partir du précédent, qui a été conservé en mémoire. 
Pour simuler la variable $N$, on prend un tel nombre $x_{j}$ et on utilise la table du paragraphe 2.I., colonne (3).

Si $f_{i-1} \leqslant x_{j}<f_{i}$, on prend $n=i$

$\left(f_{-1}=0\right)$

Pour simuler une valeur $s$ de la variable $S$, on simule $n$ fois la variable $M$ (hauteur), par la formule de la fonction inverse de la fonction exponentielle, avec un nombre pseudo-aléatoire $x_{j}$ comme argument.

$$
m_{j}=-\ln \left(\mathrm{x}-x_{j}\right)
$$

et on fait la somme $s=\sum_{j=1}^{n} m_{j}$

Les premiers couples obtenus pour $n$ (intensité) et $s$ (somme des dégâts) sont:

\begin{tabular}{c|c}
\hline$n$ & $s$ \\
\hline 0 & 0.000000 \\
0 & 0.000000 \\
2 & 2.088195 \\
0 & 0.000000 \\
$\mathrm{I}$ & $\mathrm{I} .26625 \mathrm{I}$ \\
\hline
\end{tabular}

2.3. Un seul contrat. Quelques statistiques importantes et image statistique de la fonction de répartition de la variable $S$.

$\left.I^{\circ}\right)$ Les moments et coefficients suivants ont été calculés par des moyennes portant sur 54.000 contrats. Au cours de ce calcul, on a noté les résultats correspondant à $N=9.000$, I8.000, 27.000, 36.000 et 45.000 contrats.

a) la moyenne: $\bar{S}$

b) l'écart quadratique: $v(S)=\sqrt{\overline{S^{2}}-(\bar{S})^{2}}$

c) le moment de troisième ordre:

$$
m_{3}(S)=\overline{S^{3}}-3 \overline{S^{2}} S+2(\bar{S})^{3}
$$

d) le moment de quatrième ordre:

$$
m_{4}(S)=\overline{S^{4}}-4 \overline{S^{3}} \bar{S}+6 \overline{S^{2}}(\bar{S})^{2}+2(\bar{S})^{3}
$$


e) le coefficient de symétrie: $\alpha=\frac{m_{3}(S)}{[\nu(S)]^{3}}$

f) le coefficient de curtosis: $\beta=\frac{m_{4}(S)}{[v(S)]^{4}}$

avec

$$
\bar{S}=\frac{\sum_{i=1}^{N} s_{i}}{N}, \overline{S^{2}}=\frac{\sum_{i=1}^{N} s_{i}^{2}}{N}, \overline{S^{3}}=\frac{\sum_{i-1}^{N} s_{i}^{3}}{N}, \overline{S^{4}}=\frac{\sum_{i=1}^{N} s_{i}^{4}}{N}
$$

La variable $s$ prend les $N$ valeurs données par la simulation. Le tableau ci-après reprend les résultats obtenus.

\begin{tabular}{|c|c|c|c|c|c|c|}
\hline$N$ & $\bar{S}$ & $v(S)$ & $m_{3}(S)$ & $m_{4}(S)$ & $\alpha$ & $\beta$ \\
\hline 9 & o & & & & 32 & I 7 \\
\hline I 800 & 0627 & I 2 & I. & 4 & 3 & I7 \\
\hline 2700 & o 633 & I 25 & 62 & $4 \mathrm{I}$ & $3 \mathrm{I} 3$ & I6 6232I \\
\hline 36000 & 0632 & I 249 & $6 \mathrm{I}$ & 405 & 313 & 16 65 ОI 6 \\
\hline 45000 & 06322 & I.2542 I 5 & 626 & 420 & 3173 & I6 98519 \\
\hline 54000 & o 6326388 & I 253524 & 6214783 & 4143010 & 3 I $55^{20} 3$ & I6 77970 \\
\hline
\end{tabular}

$2^{\circ}$ ) Pour $N=9000$, I $8000,27000,36000,45000$ et 54000

On a déterminé l'ımage statistique de la variable $S$ et ceci avec un pas de probabiluté de $\mathrm{O}, \mathrm{I}$.

Afin de faire apparaître le stabiluté de cette ımage en fonction du nombre d'épreuves, on a repris dans la tableau ci dessous quelques points $i$ pour les différentes valeurs de $N$.

\begin{tabular}{|c|c|c|c|c|c|c|}
\hline $2^{N}$ & 9000 & I 8000 & 27000 & 36000 & 45000 & 54000 \\
\hline I 0 & o 794333 & о 790944 & ○ 786 I I I & $\circ 7^{85361}$ & o 785622 & o $785^{8} 1_{4}$ \\
\hline 20 & o 892999 & o 892222 & o 890 I I I & 0891222 & o 891377 & o 890703 \\
\hline 30 & $\circ 94 \circ 777$ & o 941277 & o 941296 & o 942083 & o 942533 & o $94^{21} 4^{8}$ \\
\hline 40 & o 968666 & o 968333 & o 969555 & o 969666 & o 969644 & o 969499 \\
\hline 50 & o 982555 & o 9836 II & o 983666 & o 983555 & o 983177 & o $9834^{\circ} 7$ \\
\hline 60 & o 990444 & O 99 IIII & ० 991259 & o 991333 & o 99I3I I & 0991370 \\
\hline 70 & o 993999 & o 994888 & ० 995296 & o $9955^{8} 3$ & o 995333 & o $99537^{\circ}$ \\
\hline 80 & o 996222 & o 996944 & o 997222 & o 997333 & o 997 I 77 & o 997222 \\
\hline 90 & o 997999 & o 998222 & $\circ 998407$ & o 998444 & o 998288 & o 998259 \\
\hline 140 & O 999 I I I & o 998999 & O 999 I I I & o 999 I 38 & o 999022 & o 998999 \\
\hline 140 & o 999888 & o 999888 & o 999925 & о 999916 & o 9999 I r & o 999925 \\
\hline 150 & o 999999 & o 999999 & ० 999999 & o 999999 & o 999999 & o 999999 \\
\hline
\end{tabular}


On a tracé sur la figure I la représentation de cette image statistique pour $N=54000$, d'après les résultats numériques de la table de l'annexe $\mathrm{r}$.

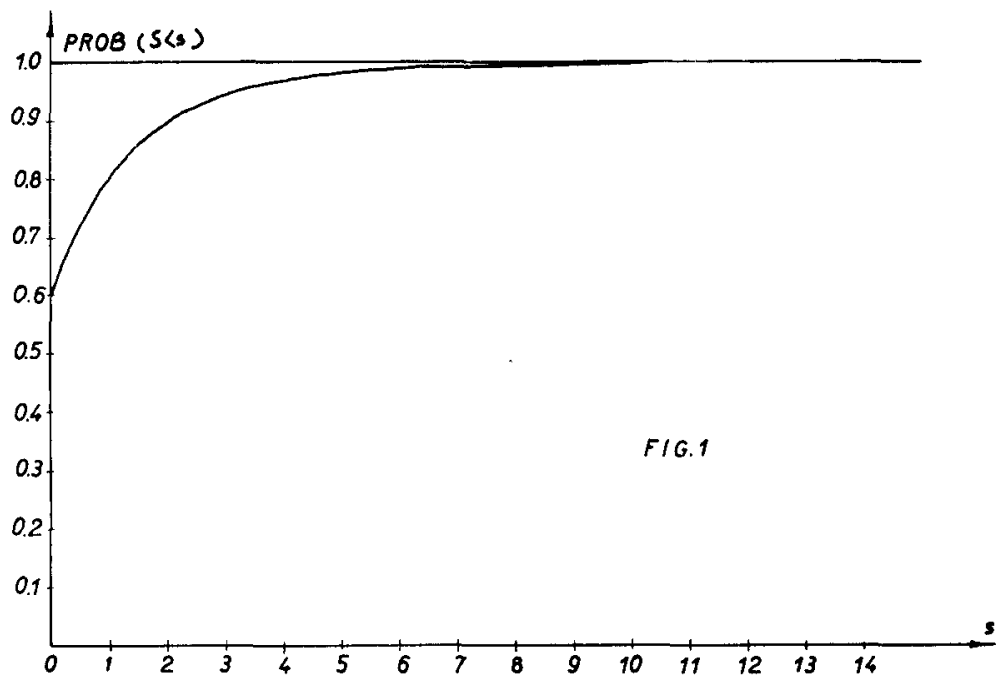

2.4. $k$ Contrats. Image statistique de la fonction de répartition de $S$. Statistiques.

A partir du ficher de base, on a construit des échantillons des variables aléatoires

$$
S_{k}=\sum_{j=1}^{k} s_{j}
$$

où $s_{j}$ est une des variables $S$ du fichier de base. Les valeurs de $k$ ont été choisies comme indiqué au tableau ci dessous:

\begin{tabular}{r|r}
\hline \multicolumn{1}{c|}{$k$} & $p$ \\
\hline 4 & 36 \\
9 & 36 \\
25 & 25 \\
100 & 9 \\
400 & 4 \\
900 & 4 \\
\hline
\end{tabular}

Une variable $S_{k}$ représente la somme des dégats pour un effectif de $k$ contrats pendant la période de référence. Pour obtenir une 
image statistique de la fonction de répartition de $S_{k}$, on a effectué Ioo simulations de cette variable. Les résultats, classés par ordre de grandeur croissante donnent une image statistique de la fonction de répartition (figure 2 , trait continu pour $k=4$ ).

En répétant la détermination expérimentale de cette image statistique, on a constaté que les échantillons successifs présentaient une dispersion assez considérable. C'est pourquoi, pour chaque valeur de $k$, on a construit $p$ échantillons (voir tableau ci dessus),

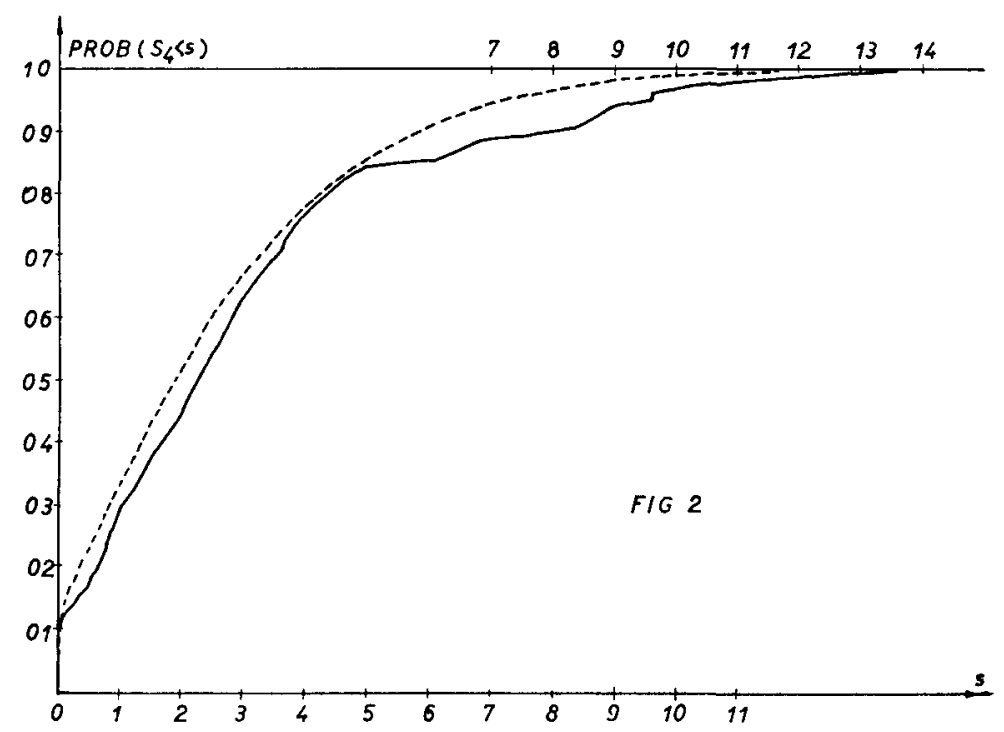

dont on a fait la moyenne arithmétique pour constituer une image statistique moyenne de la fonction de répartition de $S_{k}$. Le nombre total de contrats simulés a été imposé par les valeurs maximales de $k$ et $p$ choisies $(900 \times 4 \times$ 100 $=360000)$.

Plus précisément, on peut remarquer que les cent valeurs de $S_{k}$ qui servent à construire une image statistique sont les cent percentiles empiriques, abscisses de l'image statistique où la fréquence relative atteint un pourcentage donné. Pour chacun des percentiles, on fait la moyenne des $p$ percentilles obtenus pour obtenir le percentile de l'image moyenne. L'image statistique moyenne obtenue pour $k=4$ et $p=36$ est présentée en traits interrompus à la 
figure 2. On constate qu'elle est beaucoup plus régulière que les images statistiques dont elle est la moyenne.

Dans ce qui suit, on n'utilisera plus que les images statistiques moyennes pour résoudre les problèmes posés. En particulier, ces images statistiques moyennes ont été utilisées pour calculer les statistiques suivantes:

\begin{tabular}{rcccccc}
\hline \multicolumn{1}{c}{$k$} & $\bar{S}_{k}$ & $v\left(S_{k}\right)$ & $m_{8}\left(\bar{S}_{k}\right)$ & $m_{4}\left(S_{k}\right)$ & $\alpha\left(S_{k}\right)$ & $\beta\left(S_{k}\right)$ \\
\hline 4 & 2.5420 & 2.4703 & 20.549 & $\mathbf{1} 8 \mathrm{I} .00$ & 1.3632 & 4.8604 \\
9 & $5.700 \mathrm{I}$ & 3.6429 & 42.568 & 619.14 & 0.88054 & 3.5157 \\
25 & 16.079 & 6.2589 & 147.87 & 4849.9 & 0.60309 & 3.1604 \\
100 & 64.310 & 12.395 & 553.56 & 65890 & 0.29068 & 2.7914 \\
400 & 256.09 & $26.05 \mathrm{I}$ & 3808.0 & 1281000 & 0.21540 & 2.7815 \\
900 & 576.70 & $37.3 \mathrm{I} 8$ & 980.00 & 5450000 & 0.01886 & 2.8100 \\
\hline
\end{tabular}

L'annexe 2 reproduit la table de l'image statistique moyenne obtenue pour $k=100,400,900$.

\section{Exploitation des RÉsultats de la simulation}

Dans ce qui suit, on décrit les méthodes utilisées, et on note un certain nombre d'observations. On donne très peu de justifications à base théorique.

3.1. Comparaison des fonctions de répartition de $S_{k}$, somme des dégâts de $k$ contrats groupés, avec la fonction de répartition d'une loi de Gauss.

Le budget de $k$ contrats groupés est une somme de $k$ variables aléatoires identiques et indépendantes. On peut donc s'attendre à pouvoir appliquer les théorèmes de la tendance centrale. Pratiquement, pour un nombre $k$ donné de contrats, on peut vérifier graphiquement si l'adoption de la loi normale est admissible en utilisant la technique de la droite de Henry.

Celle-ci consiste à représenter dans un système cartésien $O x y$ les points de coordonnées $\left(x_{i}, y_{i}\right)$ tels que $x_{i}$ soit le $i$ è percentile de la loi de Gauss-Laplace réduite, et $y_{i}$ le même percentile (empirique) de la fonction de répartition particulière étudiée. Dans les graphiques des figures 3,4 et 5 , ces points n'ont été représentés que pour les percentiles de rangs multiples de 5 , et pour $k=4,9,25$, IOO, 400 et 900 . 
Si la fonction de répartition étudiée est effectivement celle d'une loi normale, et si on remplace les percentiles empiriques par les percentiles théoriques sur l'axe Oy, les points du graphique doivent se trouver sur une droite, qui est la représentation de la fonction de transformation dont il est question dans l'introduction.

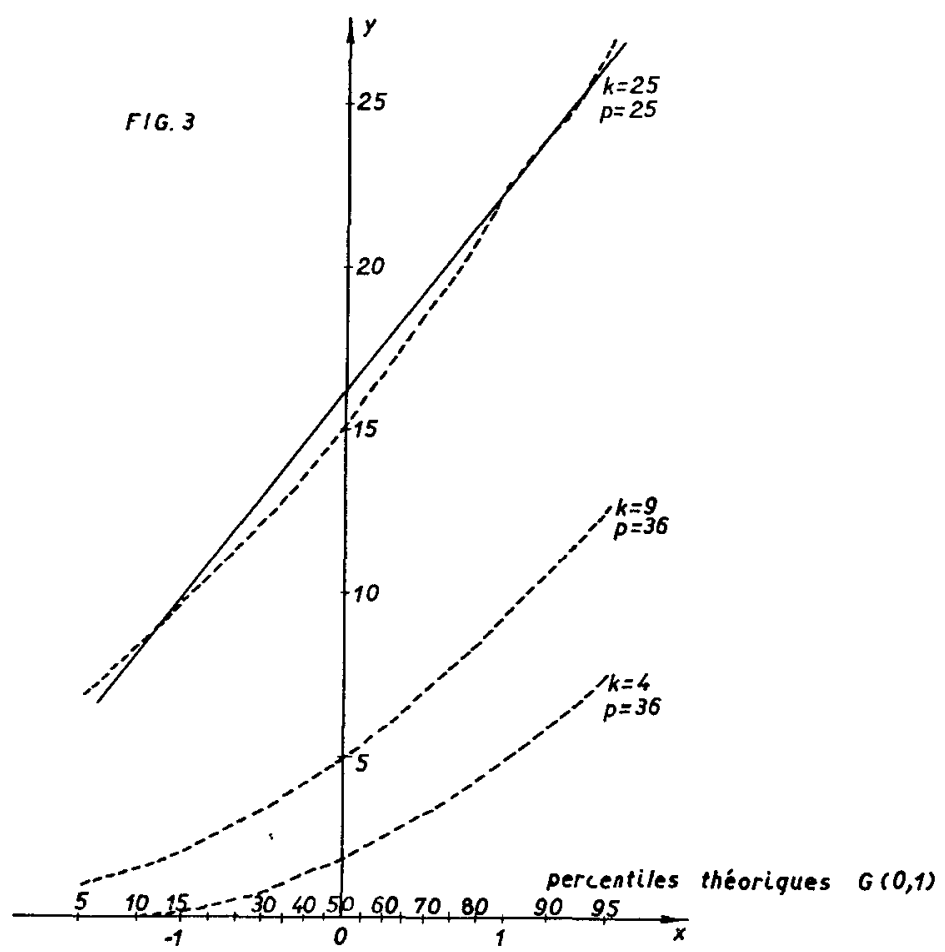

Réciproquement, la fonction de répartition est inconnue, et si les points déduits des percentiles empiriques sont suffisamment alignés, on peut admettre que la loi théorique est une loi normale. La moyenne et l'écart quadratique de cette loi normale peuvent être estimés sur le graphique (voir la fig. 4) ou encore calculés (2.3) $\mathrm{Au}$ vu des graphiques représentés, on peut admettre que pour $k=$ I00, 400, g00 la loi de la somme des dégâts est „pratiquement" normale, tandis que pour $k=4,9,25$, l'approximation de la loi réelle par une loi normale est pratiquement à rejeter.

Ceci constitue un exemple pratique de détermination de la valeur 
d'un effectif à partir duquel la tendance centrale devient pratiquement utilisable (ce que la théorie n'assure pas).

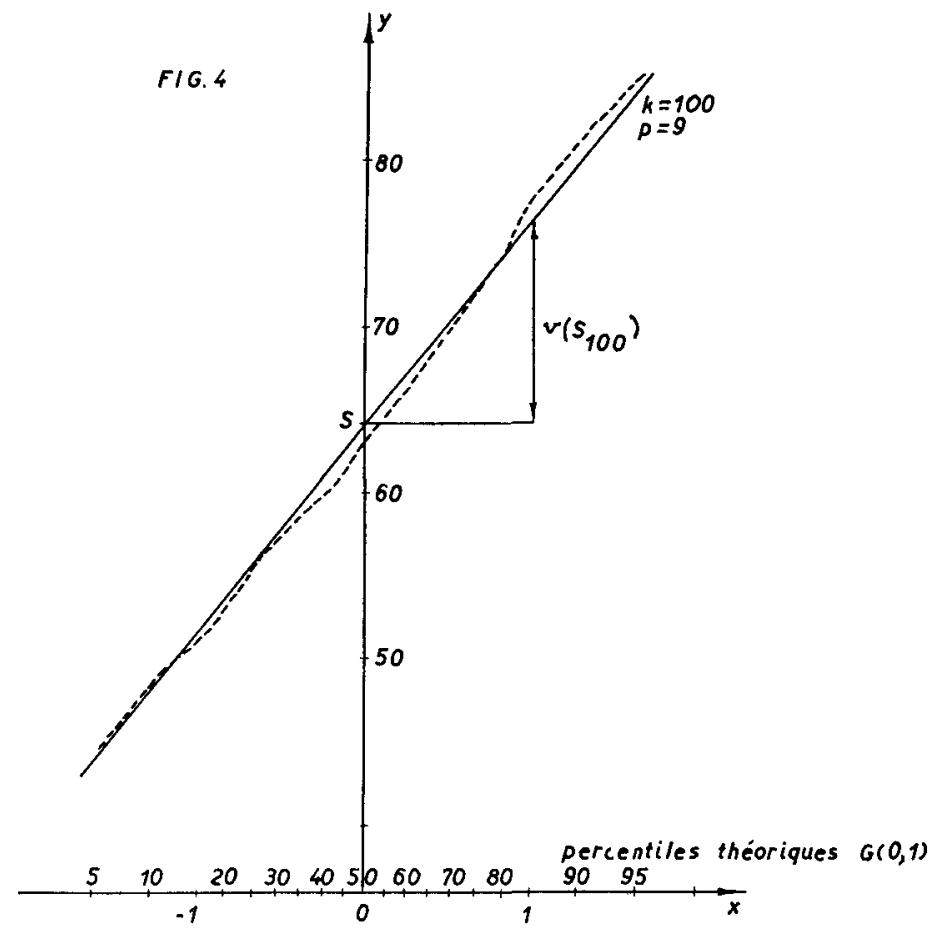

3.2. Comparaison des fonctions de répartition de la somme des dégâts de $k$ contrats groupés avec une fonction de répartition empirique de réference.

Au lieu de prendre comme loi de référence une loi de GaussLaplace, comme dans le paragraphe précédent, on peut prendre une des lois de la famille étudiée, en l'occurence la fonction de répartition empirique de la somme des dégâts pour $k=$ Ioo. On peut ainsi se dégager complètement de toute considération théorique pour la résolution des problèmes pratiques qui seront envisagés dans la suite.

Dans les graphiques des figures 6 et 7 , on a effectué un travail analogue à celui des figures 3,4 et 5 , en portant sur l'axe $O x$, au lieu des percentiles théoriques de la loi normale réduite, les percentiles empiriques de la loi de $S_{100}$, somme des dégats de roo contrats. 
A l'examen de ces graphiques, on peut admettre que la correspondance des percentiles est pratiquement linéaire entre $k=100$ d'une part, et $k=25$, 400 et 900 d'autre part. On peut écrire les

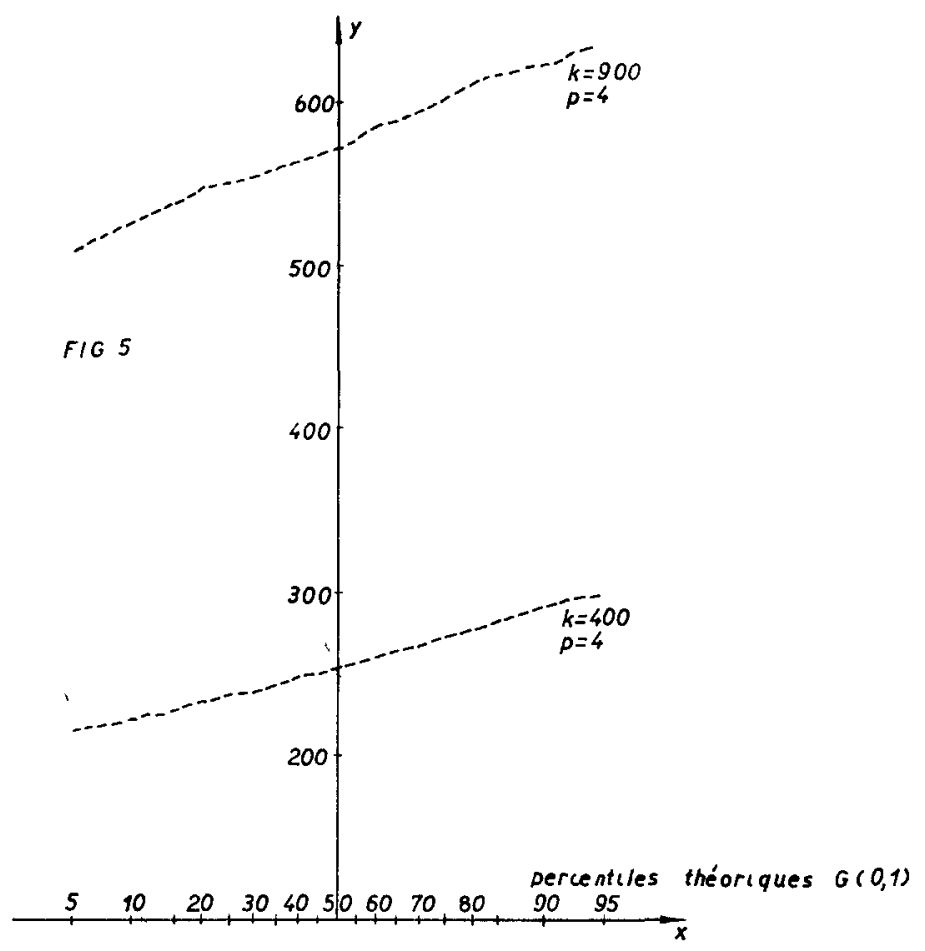

équations d'une droite qui représente cette transformation, en utilisant la méthode des moments; on choisit l'équation

$$
y=a x+b
$$

avec des coefficients $a$ et $b$ tels que les moments empiriques calculés se correspondent effectivement.

Entre $S_{100}$ et $S_{k}$ on écrit les équations

$$
\begin{aligned}
& S_{k}=a \bar{S}_{100}+b \\
& v\left(S_{k}\right)=a v\left(S_{100}\right)
\end{aligned}
$$

pour $k=25,400$ et 900 , on obtient les coefficients suivants 
IOO

L'ASSURANCE ,NON LIFE”

\begin{tabular}{r|l|c}
\hline \multicolumn{1}{c|}{$k$} & \multicolumn{1}{c|}{$a$} & \multicolumn{1}{c}{$b$} \\
\hline 25 & 0,505 & $-\mathrm{I} 6,40$ \\
400 & 2, IOI & $\mathrm{I} 2 \mathrm{I}$ \\
900 & 3, OI & 383 \\
\hline
\end{tabular}

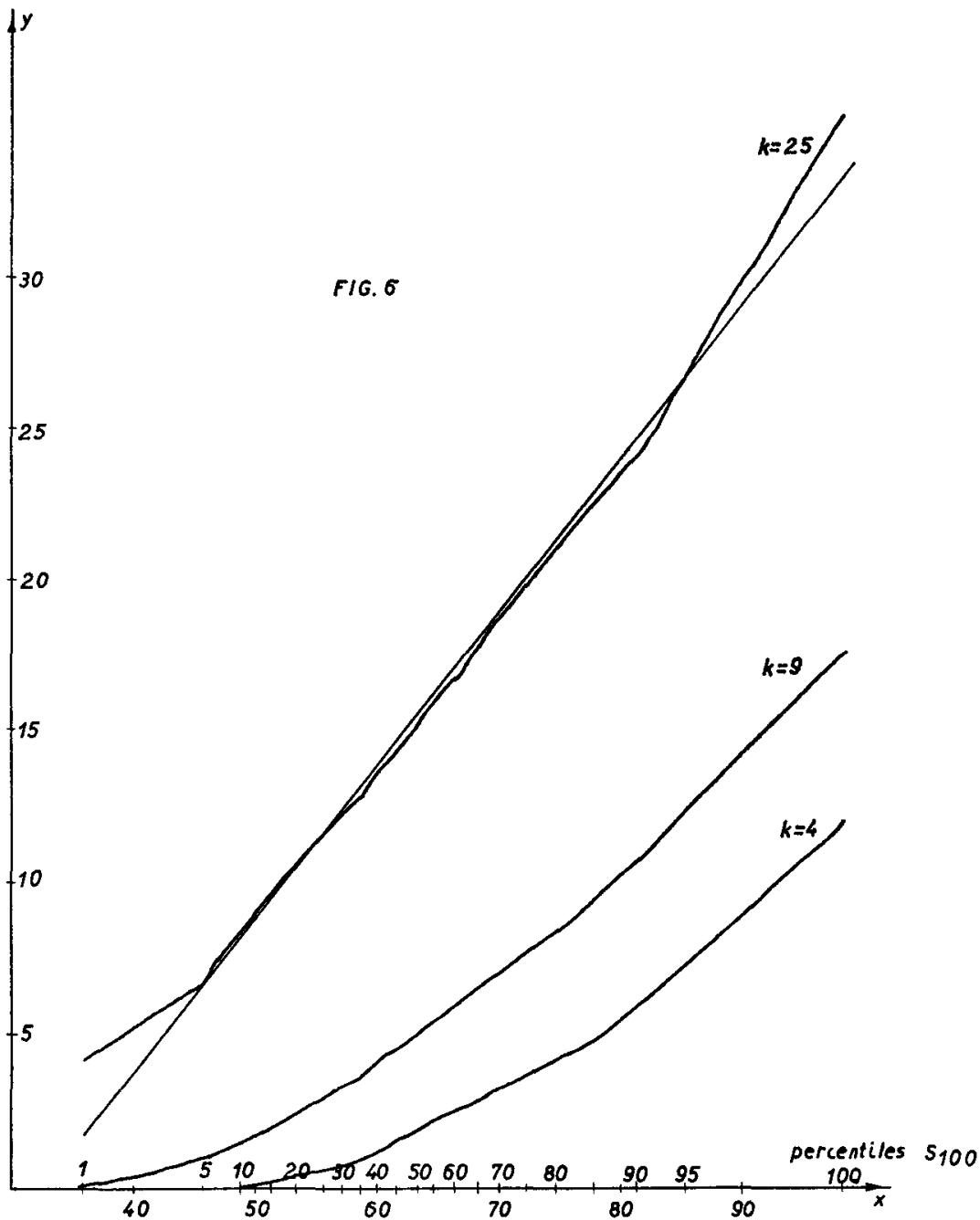


Les droites correspondantes ont été tracées sur les figures 6 et 7 Elles seront utilisées pour résoudre certains problèmes pratiques au paragraphe 3.4 .

3.3. Utilisation directe d'une image statistique moyenne

\subsubsection{Problèmes - types}

On prendra comme exemple la variable $S_{100}$, dont une table de l'image statistique est donnée en annexe 2. Pour l'utilisation directe

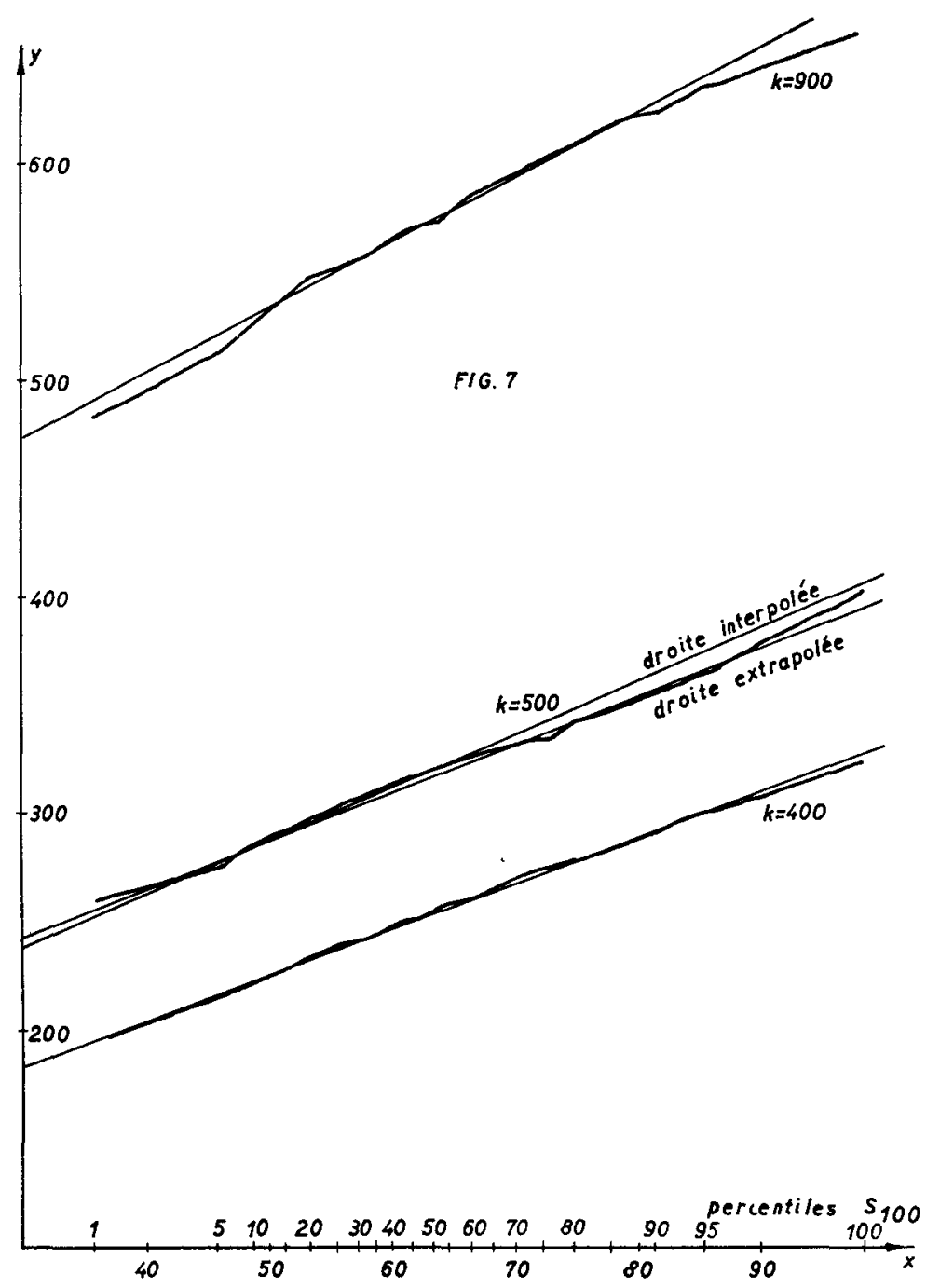


d'une telle table, on considère que chacune des valeurs apparaissant dans la table arrive avec une probabilité de o,or.

La moyenne et l'écart quadratique empiriques de $S_{100}$ sont respectivement $\bar{S}_{100}=64,3$ I et $v\left(S_{100}\right)=\mathrm{I} 2,40$.

Problème r. Estimation de la probabilité pour que la somme des dégâts relatifs à roo contrats dépasse 75 unités: 20 pour cent (ligne $8 \mathrm{I}$ de la table).

Problème 2. Estimation de la somme des dégâts qui a une probabilité d'être dépassée égale à $5 \%: 86$

$$
\text { I } \%: 98
$$

Il est à remarquer que l'estimation correspondant à une probabilité de I \% est moins précise que celle qui correspond à $5 \%$, par suite de la dispersion plus grande qui apparait dans les „,queues" des images statistiques simulées.

Problème 3. Détermination approchée (approximation de la méthode des rectangles) du montant de la prime de réassurance ,stop loss".

On suppose par exemple que lorsque la somme des dégâts dépasse 70, 74 unités pendant la période de référence (pour l'ensemble des Ioo contrats), le réassureur prend à sa charge la partie qui dépasse ce montant. Le montant 70,74 correspond à IIo \% de la valeur moyenne 64,3I. Dans ce cas, la valeur moyenne de l'intervention du réassureur est de

$\frac{(70,84-70,74)}{100}+\frac{(7 \mathrm{I}, 05-70,74)}{100}+\ldots+\frac{(98,35-70,74)}{100}=2,569$

ce qui constitue une fraction de $4 \%$ de la moyenne 64,3r. La fraction 0,04 est un taux relatif de stop loss.

3.3.2. Tables des probabilités d'écarts défavorables et de taux de stoploss

Pour les problèmes 2 et 3 du paragraphe précédent, on a effectué des calculs systématiques, en fonction d'arguments normalisés, qui sont des excès relatifs à la moyenne empirique: les annexes 3 et 4 donnent, en fonction de $\rho$ (rère colonne) et de $k$ (Ière ligne), respectivement

$$
\text { prob }\left[S_{k}>\bar{S}_{k}(\mathrm{I}+\rho)\right] \quad \text { (probabilité d'écarts défavorables) }
$$


et la valeur moyenne du taux relatif de stop loss lorsque le réassureur prend à sa charge la partie du montant $S_{k}$ qui dépasse $\bar{S}_{k}(\mathrm{I}+\rho)$

On peut retrouver dans cette dernière table le résultat calculé à la fin du paragraphe 3.3.I, et faire la constatation importante suivante: lorsque l'effectif augmente, la probabilité d'un écart défavorable et le taux relatif de stop loss diminuent pour une même valeur de p.

Ainsi, la probabilité d'un écart défavorable de 10 \% passe de 0,36 à 0,29 et à 0,06 lorsque l'effectif passe de $k=4$ à $k=$ Ioo et $k=9$ goo. Dans les mêmes conditions, le taux relatif de stop loss passe de 0,34 à 0,04 et à 0,0017 .

On remarquera cependant que la probabilité des petits écarts défavorables (jusque $20 \%$ environ) commence par croître en fonction de $k$, pour décrôte ensuite. Ceci est dû à la forte dissymétrie de la loi de $S$.

3.4. Détermination d'une fonction de répartition intermédiaire à partir de certaines fonctions de répartition simulées.

On se propose de déterminer par exemple une fonction de répartition approchée pour $S_{500}$.

3.4.1. Interpolation entre les fonctions de répartition simulées.

On peut interpoler les fonctions de répartition de $S_{100}, S_{400}$ et $S_{900}$. Pour cela, on utilise les coefficients d'interpolation de Lagrange. On note $p_{1}, p_{4}$ et $p_{9}$ les percentiles correspondants pour $k=$ IOO, 400 et 900 . Le percentile interpolé pour $k=500$ est

$$
p_{5}=L_{1} p_{1}+L_{4} p_{4}+L_{9} p_{9}
$$

avec

$$
\begin{aligned}
& L_{1}=\frac{(5-4)(5-9)}{(\mathrm{I}-4)(\mathrm{I}-9)}=\frac{-\mathrm{I}}{6}=-\mathrm{O}, \mathrm{I} 67 \\
& L_{4}=\frac{(5-\mathrm{I})(5-9)}{(4-\mathrm{I})(4-9)}=\frac{\mathrm{I} 6}{\mathrm{I} 5}=\mathrm{I}, 067 \\
& L_{9}=\frac{(5-\mathrm{I})(5-4)}{(9-\mathrm{I})(9-4)}=\frac{\mathrm{I}}{\mathrm{IO}}=\mathrm{O}, \mathrm{I}
\end{aligned}
$$


a) Interpolation entre les données brutes des trois simulations. Pour le percentile de rang Io, on trouve

$$
p_{5}=-0, \mathrm{I} 67 \times 48,76+\mathrm{I}, 067 \times 222,6+0, \mathrm{I} \times 526,8=282,0
$$

(annexe 2)

Dans ce cas, il faut disposer de trois tables de fonctions de répartition simulées, mais il n'est pas nécessaire de faire l'hypothèse de la linéarité de la transformation entre ces trois fonctions de répartition. Cette méthode pourrait servir pour interpoler entre les fonctions de répartition simulées de $S_{4}, S_{9}$ et $S_{25}$ par exemple.

b) Interpolation entre les données des simulations lissées par l'intermédiaire d'une droite de Henry (3.2.)

On prend pour $p_{1}$ les percentiles de la table de l'image statistique de la fonction de répartition de $S_{100}$, et pour $p_{4}$ et $p_{9}$ respectivement

$$
\begin{aligned}
& p_{4}=2, \text { IOI } p_{1}+\text { I } 2 \mathrm{I} \\
& p_{9}=3, \text { OI } \\
& p_{1}+383
\end{aligned}
$$

Ce sont les valeurs données par les fonctions de transformation calculées au paragraphe 3.2. On trouve ainsi pour le percentile de rang 10 ,

$$
p_{1}=48,76, \quad p_{4}=223,4, \quad p_{9}=529,8 \text { (annexe } 2 \text { et formu- }
$$
les Ia)

et, par la formule (I)

$$
p_{5}=283,2
$$

Pour appliquer cette méthode, il suffit de disposer d'une seule table (celle de $S_{100}$ ), mais il faut faire l'hypothèse de la linéarité de la transformation des fonctions de répartition.

Remarque: on peut tracer sur la figure 7 une droite de transformation de la fonction de répartition de $S_{100}$ vers $S_{500}$. Les coefficients $a$ et $b$ de celle ci s'obtiennent par interpolation entre les coefficients des droites relatives à $S_{100}$ (non tracée), $S_{400}$ et $S_{900}$. On trouve l'équation

$$
y=2,376 x+167,4
$$

Cette droite est représentée sur la figure 7 (droit interpole)é 
3.4.2. Extrapolation d'une fonction de répartition simulée

On fait l'hypothèse que les variables $S_{k}$ envisagées suivent une loi de Gauss.

a) Equation théorique d'une fonction de transformation.

On note $G(E, \sigma)$ une loi de Gauss de moyenne $E$ et écart quadratique $\sigma$. Si $S_{n 1}$ suit une loi $G\left(E_{1}, \sigma_{1}\right)$ et $S_{n 2}$ une loi $G\left(E_{2}, \sigma_{2}\right)$, avec $n \mathrm{I}=$ $k^{2} n^{2}$, on a

$$
\begin{aligned}
& E_{2}=k^{2} E_{1} \\
& \sigma_{2}=k \sigma_{1}
\end{aligned}
$$

et la droite de transformation de la fonction de répartition de $S_{n 1}$ vers celle de $S_{n^{2}}$ est

$$
y=k x+E_{1}\left(k^{2}-k\right)
$$

On trouve cette équation en exprimant que les moments de la fonction de répartition transformée doivent être $E_{2}$ et $\sigma_{2}$.

b) Transformation de $S_{100}$ en $S_{500}$.

On fait $k^{2}=5$ dans l'expression (3) et $E_{1}=63,26$ (valeur calculée à partir de la moyenne de $S$ sur 54000 contrats). On trouve

$$
y=2,236 x+\mathrm{I} 74,85
$$

La droite correspondante est représentée sur la figure 7 (droite extrapolée).

Si on l'utilise pour calculer le percentile de rang Io de $S_{500}$ à partir de celui de $S_{100}$, on trouve

$$
y=283,9
$$

Observation. La correspondance remarquable entre ce résultat et ceux du paragraphe 3.4.I présente un caractère accidentel: la droite interpolée et la droite extrapolée se coupent en effet aux environs du percentile de rang Io. On remarquera dans le tableau du paragraphe 3.4.4. que la correspondance des percentiles de rang go est moins bonne.

\subsubsection{Calcul direct de la loi de $S_{500}$.}

Dans la formule (4) on a utilisé pour $x$ les percentiles empiriques de la loi de $S_{100}$. Ayant fait l'hypothèse de la loi de Gauss, et con- 
naissant la moyenne et l'écart quadratique de $S_{100}$ à partir de ceux de $S$, déterminés au paragraphe 2.3., on aurait tout aussi bien pu prendre pour $x$ les percentiles théoriques de la loi de $S_{\mathbf{1 0 0}}$. Dans ces conditions, il est inutile de passer par l'intermédiaire de la loi de $S_{100}$, et on peut déterminer la fonction de répartition de $S_{100}$ directement à partir de celle de la loi de Gauss réduite $G(\mathrm{o}, \mathrm{I})$, de la.manière suivante:

$I^{\circ}$ Données: les moments de la loi de Delaporte sont $E(N)=0,64$ I 8 et $\sigma^{2}(N)=0,9722$

les moments de la loi exponentielle sont $E(M)=\mathrm{I}$ et $\sigma^{2}(M)=\mathrm{I}$

$2^{\circ}$ Moments de la loi de $\left.S^{1}\right)$ :

$$
\begin{aligned}
E(S) & =E(N) * E(M)=0,64 \mathrm{I} 8 \times \mathrm{I}=0,64 \mathrm{I} 8 \\
\sigma^{2}(S) & =E(N) \sigma^{2}(M)+E^{2}(M) \sigma^{2}(N) \\
& =0,64 \mathrm{I} 8 \times \mathrm{I}+\mathrm{I}^{2} \times 0,9722=\mathrm{I}, 6 \mathrm{I} 40 \\
\sigma(S) & =\sqrt{\mathrm{I}, 6 \mathrm{I} 40}=\mathrm{I}, 2704
\end{aligned}
$$

$3^{\circ}$ Moments de la loi de $S_{500}$ :

$$
\begin{aligned}
& E=0,64 \mathrm{I} 8 \times 500=320,9 \\
& \sigma=\mathrm{I}, 2704 \times \sqrt{500}=28,4 \mathrm{I}
\end{aligned}
$$

$4^{\circ}$ Fonction de transformation de $G(0,1)$ vers la loi de $S_{500}$ :

$$
y=28,4 \mathrm{I} x+320,9
$$

Application: Le percentile théorique de rang ro de la loi de Gauss réduite $G(\mathrm{O}, \mathrm{I})$ est - $\mathrm{I}, 282$. On en déduit le percentile de même rang de $S_{100}$ par la transformation

$$
y=28,4 \mathrm{r} \times(-\mathrm{I}, 282)+320,9=284,5
$$

\subsubsection{Comparaison et vérification des méthodes utilisées}

On a rassemblé ci-dessous les résultats des calculs faits précédemment pour la loi de $S_{500}$ et relatifs au percentile de rang Io.

1) L. D'Hooge et P. E. Gennart. Les risques non-viagers. Fonctions actuarielles et tables. 1965 . 
On y ajoute une colonne, où les mêmes méthodes ont été appliquées pour le percentile de rang 9o. La dernière ligne contient les mêmes résultats déduits directement de la loi empirique de $S_{500}$, dont les percentiles ont été déterminés par une moyenne de 4 épreuves $(p=4$, voir 2.4$)$. On peut considérer que la correspondance entre les résultats des diverses méthodes est très satisfaisante.

\begin{tabular}{l|c|c}
\cline { 2 - 3 } & percentile ro & percentile 90 \\
\hline Interpolation 3.4.I.a & 282,0 & 360,99 \\
Interpolation 3.4.I.b & 283,2 & 360,5 I \\
Extrapolation de $S_{100}$ & 283,9 & 356,60 \\
Calcul direct 3.4.3. & 284,5 & 357,3 \\
Simulation directe & 285,8 & 356,0 \\
\hline
\end{tabular}

\subsection{Extension}

A condition de conserver la loi de Delaporte pour l'intensité des sinistres, on peut utiliser les résultats numériques de notre étude lorsque le montant des dégâts suit une loi exponentielle de moyenne $m$ comme unité monétaire au lieu de l'unité; il suffit pour cela de multiplier par $m$ le montant des dégâts.

\subsection{Conclusions}

La simulation est une opération onéreuse. La seule constitution du fichier de base a demandé 85 heures de travail sur ordinateur IBM I620. Il faut donc éviter la simulation si le calcul peut la remplacer. Ainsi, nous avons montré que pour les calculs relatifs à la loi de $S_{500}$ l'utilisation de la loi de Gauss réduite, et des moments déduits des données du problème pouvait donner des résultats sans aucune simulation.

Cependant, la simulation a apporté deux résultats importants:

$\mathrm{I}^{\circ}$ Elle a permis de déterminer expérimentalement l'effectif à partir du quel l'approximation de la loi de Gauss est admissible (en l'occurrence, $k \geqslant$ IOo)

$2^{\circ}$ En dessous de cet effectif, elle permet d'effectuer des calculs prévisionnels sur des fonctions de répartition simulées ou interpolées.

Notons encore que les résultats de la simulatiou ont mis en évi- 
dence l'évolution des écarts défavorables et des taux relatifs de stop loss en fonction de l'effectif.

Remarque finale: dans l'exposé, on a développé à dessein les aspects les plus élémentaires, de façon à faciliter le travail de ceux qui voudraient utiliser effectivement ces méthodes.

ANNEXE I

UN SEUL CONTRAT

IMAGE STATISTIQUE DE $S$, SOMME DES DEGATS 54000 Epreuves

\begin{tabular}{|c|c|c|c|c|}
\hline \multicolumn{2}{|c|}{ Classe } & Fréquence & Fréq. Relative & Fréq. Rel. Cumulée \\
\hline De & A & & & \\
\hline .00 & .00 & 31506 & $.5^{83444}$ & $.5^{83444}$ \\
\hline .00 & . Io & $145^{2}$ & .026888 & .610333 \\
\hline .10 & .20 & I337 & .024759 & .635092 \\
\hline .20 & .30 & I3II & .024277 & $.65937^{\circ}$ \\
\hline$\cdot 30$ & .40 & I I 66 & .021592 & .680962 \\
\hline .40 & $.5^{\circ}$ & 1106 & $.0204^{8 I}$ & .701444 \\
\hline$\cdot 50$ & .60 & 1052 & $.01948 \mathrm{I}$ & .720925 \\
\hline .60 & .70 & 963 & .017833 & $\cdot 73^{8759}$ \\
\hline$\cdot 70$ & .80 & 920 & .017037 & $\cdot 755796$ \\
\hline .80 & .90 & $85 I$ & .015759 & $\cdot 771555$ \\
\hline .90 & I. 00 & $77^{\circ}$ & .014259 & $\cdot 7^{8} 5^{815}$ \\
\hline 1.00 & I. IO & 701 & $.01298 I$ & .798796 \\
\hline I. IO & I. 20 & 745 & .013796 & .812592 \\
\hline I. 20 & I. 30 & 645 & .011944 & .824537 \\
\hline$I \cdot 30$ & I. 40 & 626 & .011592 & .836129 \\
\hline I. 40 & I. $5^{\circ}$ & 621 & .011500 & .847629 \\
\hline I. 50 & I. 60 & 532 & $.00985 \mathrm{I}$ & $.8574^{8 \mathrm{I}}$ \\
\hline 1.60 & 1.70 & $5^{1} 3$ & .009500 & $.86698 \mathrm{I}$ \\
\hline $\mathrm{I} \cdot 7^{\circ}$ & 1.80 & 489 & .009055 & .876037 \\
\hline I. 80 & I.90 & $3^{87}$ & .007 I66 & .883203 \\
\hline I. 90 & 2.00 & 405 & .007500 & .890703 \\
\hline 2.00 & 2.10 & $3^{82}$ & .007074 & .897777 \\
\hline 2.10 & 2.20 & 345 & .006388 & .904166 \\
\hline 2.20 & $2 \cdot 30$ & $3^{18}$ & .005888 & .910055 \\
\hline $2 \cdot 30$ & $2 \cdot 4^{\circ}$ & 309 & .005722 & .915777 \\
\hline 2.40 & $2 \cdot 5^{\circ}$ & $28 \mathrm{I}$ & .005203 & $.92098 \mathrm{I}$ \\
\hline $2 \cdot 5^{\circ}$ & 2.60 & 280 & .005185 & .926166 \\
\hline 2.60 & 2.70 & 235 & $.00435^{I}$ & .930518 \\
\hline $2 \cdot 70$ & 2.80 & 234 & .004333 & $.934^{85} \mathrm{I}$ \\
\hline 2.80 & 2.90 & 212 & .003925 & .938777 \\
\hline 2.90 & 3.00 & 182 & $.00337^{\circ}$ & $.94^{2} \mathbf{1} 4^{8}$ \\
\hline 3.00 & 3.10 & I 88 & $.0034^{8 I}$ & .945629 \\
\hline $3 \cdot 10$ & $3 \cdot 20$ & 202 & .003740 & $.94937^{\circ}$ \\
\hline 3.20 & $3 \cdot 3^{\circ}$ & I68 & .003 III & $.95^{2481}$ \\
\hline $3 \cdot 30$ & $3 \cdot 40$ & I39 & .002574 & .955055 \\
\hline
\end{tabular}


Annexe I

UN SEUL CONTRAT

IMAGE STATISTIQUE DE $S$, SOMME DES DEGATS

54000 Epreuves

Classe Fréquence Fréq. Relative Fréq. Rel. Cumulée

De A

$3.40 \quad 3.50$

$3.50 \quad 3.60$

$3 \cdot 7^{\circ}$

3.80

3.90

4.00

4 . 10

$4 \cdot 20$

$4 \cdot 30$

$4 \cdot 40$

$4 \cdot 50$

4.60

$4 \cdot 70$

4.80

4.90

5.00

5. 10

$5 \cdot 20$

$5 \cdot 30$

$5 \cdot 4^{\circ}$

$5 \cdot 50$

5.60

$5 \cdot 7^{\circ}$

5.80

$5 \cdot 90$

6.00

I 57
I 47
I35
I 16
I 27
98
95
IOI
87
73

.002907

.957962

.002722

.960685

.002500

.963185

$.002 I_{4} 8$

.965333

$.00235 \mathrm{I}$

.967685

.001814

.969499

.001759

.971259

. 001870

.973129

. $0016 \mathrm{II}$

$.97474^{\circ}$

$.00135 \mathrm{I}$

.976092

$.0018 I_{4}$

.977907

.001148

.979055

. 001018

.980074

.001296

.981370

$.00098 \mathrm{r}$

$.98235 \mathrm{I}$

.001055

.983407

.001000

.984407

.000888

.985296

.000796

.986092

.001055

$.98714^{8}$

.000925

.988074

.000814

.988888

.000722

.98961 I

.000555

.990166

.000629

.990796

.000574

.991370

6.00

6 . 10

$.00048 \mathrm{I}$

.991851

6.10

6.20

.000685

.992537

6.20

6.30

.000425

.992962

6.30

$6.4^{\circ}$

.000518

$.99348 \mathrm{I}$

6.50

$.00035 \mathrm{I}$

.993833

6.50

6.60

6.60

6.70

.000296

.994222

.000314

.994518

6.80

6.80

6.90

.000222

.994833

$.0003 I_{4}$

.995055

7.00

$7.00 \quad 7.10$

$7.10,7.20$

$7.20 \quad 7.30$

$\begin{array}{ll}\text { II } & .000203 \\ \text { I6 } & .000296\end{array}$

.995370

.995574

.000203

$.995^{8} 70$

$7 \cdot 40$

.000185

.996074

$7 \cdot 30$

$7 \cdot 50$

ooor 85

.996259

.996444

$7 \cdot 50$

7.60

.000129

.996574 


\section{ANNEXe I \\ UN SEUL CONTRAT \\ IMAGE STATISTIQUE DE $S$, SOMME DE DEGATS 54000 Epreuves}

Classe $\mathrm{De}$

$7 \cdot 60$

$7 \cdot 70$

7.80

$7 \cdot 90$

8.00

8. 10

8.20

$8 \cdot 30$

8.40

8.50

8.60

8.70

8.80

8.90

9.00

9 . I0

9.20

$9 \cdot 30$

9.40

9.50

9.60

9.70

9.80

9.90

Io. 00

Io. 10

I0. 20

ro. 30

Io. 40

Io. 50

Io. 60

ro. 70

I0. 80

Io. 90

I I . 00

I I . IO

I I . 20

I I. 30

II .40

I I . 50

II. 60

I I . 70
A

$7 \cdot 7^{\circ}$

7.80

7.90

8.00

8 . 10

8.20

8.30

8.40

$8.5^{\circ}$

8.60

8.70

8.80

8.90

9.00

9.10

9.20

$9.3^{\circ}$

$9 \cdot 4^{\circ}$

9.50

9.60

9.70

9.80

9.90

I0.00

I0. Io

10. 20

Io. 30

Io. 40

Io. $5^{\circ}$

10.60

I0. 70

Io. 80

I0. 90

I I . 00

I I . IO

I I . 20

I I 30

II 40

I I $5^{\circ}$

II . 60

II 70

II 80
Fréquence

Fréq. Relative
Fréq. Rel. Cumulée $.99674^{\circ}$

.997018

.997074

.997222

.997333

$.9974^{\circ} 7$

.997481

.997648

.997777

.997888

.997981

.998074

.998 I 85

.998259

.998314

.998370

.998462

.998574

.998666

$.99874^{\circ}$

.998777

.998888

.998944

.998999

.999092

.999277

.999296

.999370

.999425

.999444

.999499

.999537

.999537

.999574

.999629

.999629

.999648

.999648

.999666

.999685

.999722

.999722 


\section{ANnexe I \\ UN SEUL CONTRAT \\ IMAGE STATISTIQUE DE $S$, SOMME DES DEGATS}

54000 Epreuves

Classe

Fréquence Freq. Relative

Fréq. Rel. Cumulée

De

A

$\begin{array}{ll}\text { II. } 80 & 11.90 \\ \text { I1.90 } & 12.00 \\ 12.00 & 12.10 \\ 12.10 & 12.20 \\ 12.20 & 12.30 \\ 12.30 & 12.40 \\ 12.40 & 12.50 \\ 12.50 & 12.60 \\ 12.60 & 12.70 \\ 12.70 & 12.80 \\ 12.80 & 12.90 \\ 12.90 & 13.00 \\ 13.00 & 13.10 \\ 13.10 & 13.20 \\ 13.20 & 13.30 \\ 13.30 & 13.40 \\ 13.40 & 13.50 \\ 13.50 & 13.60 \\ 13.60 & 13.70 \\ 13.70 & 13.80 \\ 13.80 & 13.90 \\ 13.90 & 14.00 \\ 14.00 & 14.10 \\ 14.20 & 14.20 \\ 14.20 & 14.30 \\ 14.30 & 14.40 \\ 14.40 & 14.50 \\ 14.50 & 14.60 \\ 14.60 & 14.70 \\ 14.70 & 14.80 \\ 14.80 & 14.90 \\ 14.90 & 15.00\end{array}$

\begin{tabular}{|c|c|c|}
\hline 0 & .000000 & .999722 \\
\hline o & .000000 & .999722 \\
\hline 4 & .000074 & .999796 \\
\hline I & .000018 & $.9998 r_{4}$ \\
\hline o & .000000 & $.9998 I_{4}$ \\
\hline $\mathbf{o}$ & .000000 & $.9998 I_{4}$ \\
\hline I & .000018 & .999803 \\
\hline o & .000000 & .999833 \\
\hline o & .000000 & .999833 \\
\hline o & .000000 & .999833 \\
\hline $\mathbf{o}$ & .000000 & .999833 \\
\hline o & .000000 & .999833 \\
\hline 0 & .000000 & .999833 \\
\hline 0 & .000000 & . 999833 \\
\hline I & .000019 & $.99985 \mathrm{I}$ \\
\hline $\mathbf{o}$ & .000000 & $.99985^{\mathrm{I}}$ \\
\hline I & .000018 & .999870 \\
\hline I & .000018 & .999888 \\
\hline I & .000018 & .999907 \\
\hline 0 & .000000 & .999907 \\
\hline I & .000018 & .999925 \\
\hline o & .000000 & .999925 \\
\hline 0 & .000000 & .999925 \\
\hline$\circ$ & .000000 & .999925 \\
\hline 2 & .000037 & .999962 \\
\hline I & .000018 & .99998I \\
\hline I & .000018 & .999999 \\
\hline 0 & .000000 & .999999 \\
\hline o & .000000 & .999999 \\
\hline 0 & .000000 & .999999 \\
\hline o & .000000 & .999999 \\
\hline 0 & .000000 & . 999999 \\
\hline
\end{tabular}

Annexe 2

\section{MOYENNE IMAGE STATISTIQUE}

$\begin{array}{cccc} & P=9 & P=4 & P=4 \\ & K=100 & K=400 & K=900 \\ \mathrm{I} & 35.810937 & 194.381850 & 483.857800 \\ 2 & 40.457026 & 203.158040 & 494.814620 \\ 3 & 42.173830 & 208.227450 & 501.108070 \\ 4 & 43.205140 & 212.399570 & 507.327900\end{array}$


ANNEXe 2

MOYENNE IMAGE STATISTIQUE

$$
\begin{aligned}
& P=9 \\
& K=\text { гоо }
\end{aligned}
$$

$44 \cdot 443374$

$45 \cdot 755^{1} \mathrm{I}_{3}$

46.688143

47.433847

48.098 I I 2

48.756672

$49 \cdot 306315$

49.829517

$50.39277^{\circ}$

50.794502

$5 \mathrm{I} \cdot 344973$

51.799667

52.081677

$5^{2} \cdot 367562$

52.968056

$53 \cdot 318453$

53.879375

54.234845

54.815687

$55 \cdot 19774^{8}$

55.556980

$55 \cdot 75264 \mathrm{I}$

56.127717

$5^{6.5}$ I 7837

57.050903

$57 \cdot 309046$

$57 \cdot 467345$

$57 \cdot 760184$

57.934370

58.379060

$5^{8.695560}$

59.062500

$59 \cdot 302428$

59.515447

59.801793

60.031300

$60.3035^{12}$

60.560064

60.902626

$6 \mathrm{I} .213005$

61.660194

61.909792

62.218021

62.454640

62.688465
$P=4$
$K=400$

$2 \mathrm{I} 5.095620$

216.949550

217.610520

$219 \cdot 310810$

221 . 124630

$222.6074^{80}$

$224 \cdot 537220$

225.944640

226.525470

$227 \cdot 392340$

228 . 709 I 50

229.923850

231.222370

232.355230

232.999740

233.248880

$233 \cdot 7965$ 10

234.418390

$235.93277^{\circ}$

237.020190

$237 \cdot 320640$

$237 \cdot 782560$

$23^{8} \cdot 514690$

239.3323 10

239.920730

240.630640

24 I 454390

242.213080

243 . 125600

243.245600

$244 \cdot 454320$

$245 \cdot 442010$

$246.337^{1} 5^{\circ}$

246.449030

247.437770

248 . 124280

248.270330

248.559440

249.243910

249.942670

250.677120

$25 \mathrm{I} .62 \mathrm{II} 2 \mathrm{O}$

$252.274^{100}$

252.634200

253.558800
$P=4$

$K=900$

$5 \mathrm{II} \cdot 736470$

$5^{17} \cdot 45^{8950}$

520.147000

522.469820

$523 \cdot 3^{84920}$

526.786120

527.900600

529.955100

$534.87 \times 270$

$537 \cdot 34$ I 370

538.542650

$54 \mathrm{I} \cdot 722900$

542.395400

$543 \cdot 498020$

$545 \cdot 72375^{\circ}$

547.052320

$54^{8} .37477^{\circ}$

548.998620

549.940170

$55^{\circ} .96597^{\circ}$

$55^{\mathrm{I}} \cdot 34347^{\circ}$

$55 \mathrm{I} .96 \mathrm{I} 300$

$55^{2} .83737^{\circ}$

$553.977^{\circ} 7^{\circ}$

$555 \cdot 335500$

$555 \cdot 55^{24} 5^{\circ}$

$55^{8}$. 16937 o

$559.45^{2}$ I 70

560.463720

$560.92557^{\circ}$

561.247320

562.321850

$563 \cdot 366500$

564.506320

565 . 5487 o

$565 \cdot 7^{1} 595^{\circ}$

$566.68855^{\circ}$

567.160650

567.557900

$568.04455^{\circ}$

569.718900

$569.98195^{\circ}$

570.986100

$57 \mathrm{I} \cdot 470700$

572.181720 
ANNEXE 2

MOYENNE IMAGE STATISTIQUE

$P=9$

$K=$ I00

63.281537

$63 \cdot 44^{21} 67$

63.780960

$64 \cdot 1553^{8} 5$

$64 \cdot 36$ I 370

$64 \cdot 59676 \mathrm{I}$

64.986747

$65 \cdot 312670$

$65 \cdot 573^{8} 35$

66.064563

66.409206

66.811998

67.167274

$67 \cdot 5^{8} 575^{6}$

$67.87054^{\circ}$

68.235498

68.660640

69.087292

69.484280

69.724957

$70.1014 \mathrm{II}$

70.462124

70.842231

7 r. 054620

7 I. 394507

72.221943

72.562167

73.005240

73.981650

$74 \cdot 365360$

$74 \cdot 730391$

75.221394

75.990143

76.529105

77.062385

77.7929I 3

78.161836

78.643232

79.271936

80.366588

81.280561

82.014604

82.335445

83.172634

84.217065
$P=4$

$K=400$

$254 \cdot 364600$

$254.85415^{\circ}$

$255.475^{6} 5^{\circ}$

$25^{6}$. I $7205^{\circ}$

256.979270

257.879070

258.825150

259.076050

259.587920

$259.97347^{\circ}$

260.85 I 450

261.286420

$26 \mathrm{I} \cdot 77775^{\circ}$

$262.27925^{\circ}$

263.571520

$264 \cdot 769520$

$265 \cdot 318700$

266.968570

$267 \cdot 368770$

267.954070

268.842850

269.845420

270.402900

271.172420

271.760400

273.256020

274.205970

274.817150

$275 \cdot 543600$

276.939600

277.878170

279 . $14245^{\circ}$

280.328420

$281.02765^{\circ}$

28 I . 464370

282.493050

283.778820

286.053600

288. 122000

289.826050

$292.6275^{20}$

$294.12235^{\circ}$

$295.03735^{\circ}$

295.996720

296.820050
$P=4$

$K=900$

$573 \cdot 246400$

$575 \cdot 778500$

577.061800

577.682500

578.308150

578.827900

$580.235^{8} 70$

$5^{81}$. $16505^{\circ}$

$5^{82} \cdot 395^{100}$

583.981670

586 . 163400

586.980200

$587 \cdot 568300$

588.269150

588.996200

590.505070

$59 \mathrm{I} \cdot 35335^{\circ}$

592.198700

592.728650

$593.66795^{\circ}$

594.862800

595.904020

597.610970

599.834170

601.919970

602.954800

$604 \cdot 942720$

605.311570

606.776520

$607.50275^{\circ}$

$609 \cdot 338620$

6 II. 430430

613.053670

$6 \mathrm{I} 4 \cdot 7 \mathrm{OI} 75^{\circ}$

$615 \cdot 174820$

618.008920

$6 \mathrm{r} 8.507750$

619.402400

$619.65177^{\circ}$

$621.163^{8} 70$

623.272720

$625 \cdot 710100$

628 . I I 4620

$629.48425^{\circ}$

631.224600 
ANNEXE 2

\section{MOYENNE IMAGE STATISTIQUE}

$\begin{array}{rlll} & P=9 & P=4 & P=4 \\ & K=\text { I00 } & K=400 & K=900 \\ 95 & 85.329858 & 298.403600 & 635.040100 \\ 96 & 86.798047 & 301.986470 & 639.84737^{\circ} \\ 97 & 88.541006 & 305.571350 & 647.587120 \\ 98 & 89.685274 & 312.246570 & 654.257820 \\ 99 & 93.553544 & 317.582720 & 658.976400 \\ 100 & 98.349090 & 323.612220 & 668.641270\end{array}$

AnNexe 3

PROBABILITES D' ECARTS DEFAVORABLES

$K=4 K=9 K=25 \quad K=$ I00 $K=400 \quad K=900$

\begin{tabular}{|c|c|c|c|c|c|c|}
\hline .00 & $\cdot 39$ & .43 & .45 & .47 & $.4^{8}$ & .49 \\
\hline $.0 I$ & .39 & .42 & .44 & .45 & .45 & .42 \\
\hline .02 & $\cdot 3^{8}$ & .42 & .43 & .42 & .40 & $\cdot 3^{8}$ \\
\hline .03 & $\cdot 3^{8}$ & $.4 \mathrm{I}$ & .42 & $.4 \mathrm{I}$ & .36 & $\cdot 3 \mathrm{I}$ \\
\hline .04 & $\cdot 3^{8}$ & $\cdot 4 \mathrm{I}$ & $.4 \mathrm{I}$ & .39 & .34 & .28 \\
\hline .05 & $\cdot 37$ & $.4^{\circ}$ & .40 & $\cdot 38$ & .30 & .23 \\
\hline .06 & $\cdot 37$ & .39 & .39 & $\cdot 36$ & .27 & .20 \\
\hline .07 & .37 & .39 & $\cdot 38$ & $\cdot 34$ & .25 & .16 \\
\hline .08 & $\cdot 36$ & $\cdot 38$ & $\cdot 37$ & .33 & .22 & . I I \\
\hline .09 & $\cdot 36$ & $\cdot 38$ & .37 & $\cdot 3 I$ & .20 & .08 \\
\hline . 10 & $\cdot 36$ & $\cdot 3^{8}$ & $\cdot 36$ & .29 & .16 & .06 \\
\hline . II & $\cdot 35$ & $\cdot 37$ & $\cdot 35$ & .27 & .14 & .04 \\
\hline .12 & $\cdot 35$ & $\cdot 3^{6}$ & .35 & .26 & .13 & .04 \\
\hline . I3 & .35 & $\cdot 3^{6}$ & $\cdot 34$ & .24 & .12 & .03 \\
\hline .14 & $\cdot 35$ & $\cdot 35$ & $\cdot 33$ & .23 & . I I & .02 \\
\hline . I 5 & $\cdot 34$ & $\cdot 35$ & $\cdot 32$ & .23 & .09 & 0.00 \\
\hline .16 & $\cdot 34$ & $\cdot 34$ & $\cdot 31$ & $.2 \mathrm{I}$ & .06 & \\
\hline .17 & $\cdot 34$ & $\cdot 34$ & $\cdot 30$ & .19 & .05 & \\
\hline .18 & .33 & $\cdot 34$ & .29 & .19 & $.0_{4}$ & \\
\hline . I9. & .33 & $\cdot 33$ & .29 & . 18 & .03 & \\
\hline .20 & $\cdot 33$ & $\cdot 32$ & .28 & . I6 & .03 & \\
\hline $.2 \mathrm{I}$ & $\cdot 32$ & $\cdot 32$ & .27 & .15 & .03 & \\
\hline .22 & $\cdot 32$ & $\cdot 3 I$ & .27 &.$I_{4}$ & .02 & \\
\hline .23 & .32 & $\cdot 3 I$ & .26 & .13 & .02 & \\
\hline .24 & $\cdot 3 \mathbf{I}$ & $\cdot 3^{I}$ & .25 & .12 & .02 & \\
\hline .25 & $\cdot 3 \mathbf{I}$ & .30 & .24 & I I I & 0.00 & \\
\hline .26 & $\cdot 3 \mathbf{I}$ & $3^{\circ}$ & .24 & I I & & \\
\hline .27 & .30 & .29 & .23 & . IO & & \\
\hline .28 & .30 & .29 & .22 & .09 & & \\
\hline .29 & 30 & .28 & .22 & .08 & & \\
\hline$\cdot 30$ & .29 & .27 & $.2 \mathrm{I}$ & .07 & & \\
\hline$\cdot 3 \mathrm{I}$ & .29 & .27 & .20 & .06 & & \\
\hline$\cdot 32$ & .29 & .27 & .20 & .06 & & \\
\hline$\cdot 33$ & .29 & .26 & .19 & .05 & & \\
\hline
\end{tabular}




\section{Annexe 3}

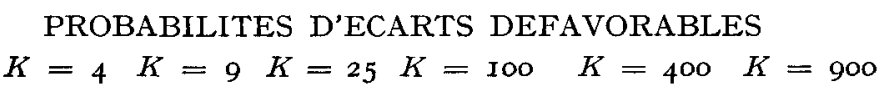

\begin{tabular}{|c|c|c|c|c|}
\hline .34 & .28 & .26 & .19 & .05 \\
\hline .35 & .28 & .26 & . I9 & .04 \\
\hline$\cdot 36$ & .28 & .25 & . I 8 & .04 \\
\hline$\cdot 37$ & .28 & .25 & .18 & .04 \\
\hline$\cdot 3^{8}$ & .27 & .24 & . I 7 & .03 \\
\hline .39 & .27 & .24 & . I6 & .03 \\
\hline .40 & .27 & .24 & .16 & .02 \\
\hline $.4 \mathrm{I}$ & .27 & .23 &.${ }_{5}$ & .02 \\
\hline .42 & .26 & .23 & . I 5 & .02 \\
\hline .43 & .26 & .22 &. $\mathrm{I}_{4}$ & .02 \\
\hline$\cdot 44$ & .26 & .22 &.$I_{4}$ & .02 \\
\hline .45 & .26 & .22 & .13 & .02 \\
\hline .46 & .25 & .21 & . I3 & 0.00 \\
\hline .47 & .25 & .21 & . I 2 & \\
\hline .48 & .25 & .20 & .12 & \\
\hline .49 & .25 & .20 & . I I & \\
\hline . 50 & .24 & . I9 & . II & \\
\hline .55 & .23 & . 18 & .09 & \\
\hline .60 & .22 & .17 & .07 & \\
\hline .65 & .21 & .16 & .06 & \\
\hline$\cdot 70$ & .20 & .15 & .05 & \\
\hline$\cdot 75$ & . I9 & . I3 & .04 & \\
\hline .80 & .18 & . I 2 & .04 & \\
\hline .85 & .17 & . II & .03 & \\
\hline .90 & . I6 & . I0 & .03 & \\
\hline .95 & .15 & .09 & .02 & \\
\hline I. OO &.${ }^{4} 4$ & .08 & .02 & \\
\hline I.05 &.$I_{4}$ & .08 & & \\
\hline 1.10 & . I3 & .07 & & \\
\hline I. I 5 & .12 & .06 & & \\
\hline I . 20 & .12 & .06 & & \\
\hline 1.25 & . I I & .05 & & \\
\hline $1 \cdot 30$ & . I I & .04 & & \\
\hline I $\cdot 35$ & . I0 & .04 & & \\
\hline I. $4^{\circ}$ & .10 & .03 & & \\
\hline I. 45 & .09 & .03 & & \\
\hline I. 50 & .09 & .03 & & \\
\hline I. 60 & .08 & .02 & & \\
\hline $\begin{array}{l}\text { I. } 70 \\
\text { I. } 80\end{array}$ & $\begin{array}{l}.07 \\
.06\end{array}$ & .02 & & \\
\hline I. 90 & .05 & & & \\
\hline 2.00 & .05 & & & \\
\hline 2. IO & .04 & & & \\
\hline 2.20 & .04 & & & \\
\hline $2 \cdot 30$ & .03 & & & \\
\hline 2.40 & .03 & & & \\
\hline
\end{tabular}




\section{AnNexe 3}

PROBABILITES D' ECARTS DEFAVORABLES

$$
\begin{array}{ll} 
& K=4 \quad K=9 K=25 \quad K=100 \quad K=400 \quad K=900 \\
2.50 & .03 \\
2.60 & .02 \\
2.70 & .02
\end{array}
$$$$
2.60 \quad .02
$$$$
2.80 \quad .02
$$

\begin{tabular}{|c|c|c|c|c|c|c|}
\hline & $K=4$ & $K=9$ & $K=25$ & $K=4^{\circ 00}$ & $K=400$ & $K=900$ \\
\hline .00 & .3765310 & .2543780 & I 556800 & .0775030 & .0409380 & .0260120 \\
\hline .02 & $\cdot 3687800$ & .2459 I 50 & .1468960 & .0685930 & .0321320 & .0174290 \\
\hline .04 & 36 I I 800 & .2376900 & .1385180 & .0604040 & .0248730 & . ol J 0690 \\
\hline .06 & .3537220 & .2296910 & . I 304990 & .0528960 & .0187600 & .0063680 \\
\hline .08 & .3463650 & .2218910 & .1228360 & .0460660 & $.013^{8} 790$ & .0033110 \\
\hline .10 & $\cdot 339165^{\circ}$ & .2142910 & . II 54460 & .0399620 & .0100020 & .0017010 \\
\hline .12 & .3320760 & .2069000 & .1083520 & .0345960 & .007 I I 90 & .0007950 \\
\hline .14 & $\cdot 3^{2} 50760$ & .19974 Io & IOI 5860 & .0297300 & $.00475^{10}$ & .0002210 \\
\hline .16 & $\cdot 3182670$ & .1927430 & $.095^{1} 44^{0}$ & .0252630 & .0029690 & \\
\hline .18 & $\cdot 3115480$ & .1859430 & .0890720 & $.021345^{\circ}$ & .0019240 & \\
\hline .20 & $\cdot 3049480$ & . I793500 & .0832960 & $.017^{8} 460$ & .0012310 & \\
\hline .22 & .2984690 & .1729610 & .0778240 & .0147950 & .0006380 & \\
\hline .24 & .2921050 & .1667610 & .0726640 & .0122400 & $.00023^{80}$ & \\
\hline .26 & .2859050 & .1607180 & .0677780 & $.009943^{\circ}$ & & \\
\hline .28 & .2798300 & . I 548660 & .0631950 & .0079510 & & \\
\hline$\cdot 30$ & $.273^{8} 54^{\circ}$ & . I 492660 & .0588300 & .0064150 & & \\
\hline$\cdot 3^{2}$ & $.268054^{\circ}$ & . I $43^{8660}$ & $.054737^{\circ}$ & $.005 \mathrm{rI} 90$ & & \\
\hline$\cdot 34$ & $.26227^{80}$ & . 1385820 & .0508430 & $.00405 \mathrm{IO}$ & & \\
\hline$\cdot 36$ & .2566780 & .1334460 & .0471400 & $.003154^{\circ}$ & & \\
\hline$\cdot 3^{8}$ & $.251175^{\circ}$ & .1284680 & .0436230 & .0023860 & & \\
\hline .40 & $.245775^{\circ}$ & .1236680 & .0403420 & .0018400 & & \\
\hline .42 & .2404620 & .1190390 & $.037^{29} 4^{\circ}$ & $.00144^{\circ 0}$ & & \\
\hline .44 & .2352620 & . I I $4554^{\circ}$ & .0344200 & .0010400 & & \\
\hline .46 & .2301490 & . IIO25IO & .0317680 & .0000000 & & \\
\hline $.4^{8}$ & $.225 \times 490$ & . $106064^{\circ}$ & .0292960 & & & \\
\hline .50 & .2201980 & .1020670 & .0270790 & & & \\
\hline $.5^{2}$ & .2153980 & .0982670 & $.025035^{\circ}$ & & & \\
\hline .54 & .2106540 & .0944720 & .0230350 & & & \\
\hline .56 & .2060540 & .0908720 & $.021225^{\circ}$ & & & \\
\hline $.5^{8}$ & .2015360 & .08733 七o & .0195400 & & & \\
\hline .60 & . I97 I 360 & .0839310 & .0179700 & & & \\
\hline .62 & .1927710 & .0806210 & .0165700 & & & \\
\hline .64 & . I8857 IO & .0774210 & $.015^{2240}$ & & & \\
\hline .66 & I843710 & .0742210 & $.014024^{\circ}$ & & & \\
\hline .68 & .1803410 & .0712180 & .0128880 & & & \\
\hline .70 & .1763410 & .0682180 & .0118880 & & & \\
\hline
\end{tabular}

Annexe 4

TAUX RELATIF DE STOP LOSS 
ANNEXE 4

TAUX RELATIF DE STOP LOSS

$\begin{array}{lccc} & K=4 & K=9 & K=25 \\ .75 & .1667070 & .0612830 & .0094120 \\ .80 & .1575780 & .0549650 & .0074120 \\ .85 & .1488900 & .0490940 & .0058120 \\ .90 & .1407160 & .0438410 & .0043120 \\ .95 & .1328840 & .0389410 & .0032490 \\ 1.00 & .1254980 & .0345350 & .0022490 \\ 1.05 & .1184980 & .0305350 & \\ 1.10 & .1119000 & .0269620 & \\ 1.15 & .1054540 & .0237110 & \\ 1.20 & .0994540 & .0207110 & \\ 1.30 & .0880160 & .0158580 & \\ 1.40 & .0778030 & .0120360 & \\ 1.50 & .0687040 & .0090360 & \\ 1.60 & .0605590 & .0068400 & \\ 1.70 & .0534050 & .0048400 & \\ 1.80 & .0471580 & & \\ 1.90 & .0411620 & & \\ 2.00 & .0361620 & & \\ 2.20 & .0273440 & & \\ 2.40 & .0204040 & & \\ 2.60 & .0151180 & & \\ 2.80 & .0111180 & & \\ 2.98 & 0.0000000 & \end{array}$

